\title{
Long COVID-19: Rehabilitative and Restorative Challenges
}

\author{
Atul Bhatnagar
}

International Journal of Prosthodontics and Restorative Dentistry (2021): 10.5005/jp-journals-10019-1316

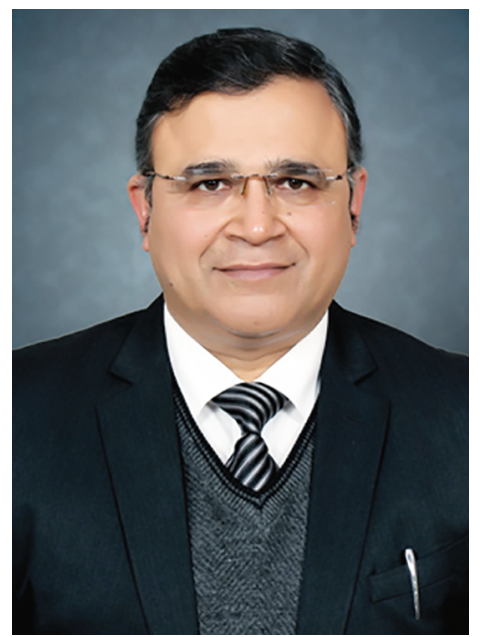

Atul Bhatnagar
WHO declared COVID-19 a pandemic on March 11, 2020. Even after 17 months, 206 million cases with 4.3 million deaths, there is the arrival of fresh waves after the initial one and the emergence of viral variants. ${ }^{1}$ The pandemic is not showing any sign of waning.

The long COVID has meant upheaval in dental and health spheres alike, worldwide. The frequent lockdowns with guidelines and protocols to prevent the spread of infection are in place. This has created an unprecedented disruption in routine dental health services. Dental procedures are being limited to dental emergencies. ${ }^{2}$

As dental health services are/will be gradually opening up to the public and keeping in tune with current COVID recommendations and guidelines, several challenges loom large. There is a huge backlog of cases with restorative and rehabilitative needs. ${ }^{3}$ With emphasis during the current pandemic on fewer and shorter procedural appointments meeting the routine patients' backlog seems an uphill task. Patients with post-COVID dental issues are reporting as new arrivals with increased frequency and urgency. Commonly reported conditions in such patients include bruxism, clenching, chipped tooth, cracked tooth syndrome, TMD symptoms, bleeding gums, dry mouth, and mucormycosis and in its aftermath, severely mutilating maxillofacial defects. ${ }^{4,5}$

Furthermore, geriatric patients as well as those with underlying medical conditions are a susceptible group for contracting COVID19 infection. ${ }^{6}$ However, with the rapid pace of vaccination roll-out, the vulnerability of this group of patients is being minimized.

The adoption of strategies that may prove to be prudent and efficient at meeting the COVID-19 challenges is much needed. Any such strategy needs to be mindful of being both preventive and less invasive. ${ }^{7}$ Prior teleconsultation, telediagnosis, emergency, and dental procedure triaging may reduce the actual chairside time for both patient and the restorative/rehabilitative dental team. ${ }^{8}$ Adoption of minimally invasive adhesive dentistry along laser and digital dentistry may eliminate aerosol-generating scenario. ${ }^{6}$ Removable dentures provision for partially edentulous mouths and maxillofacial prosthetic defect(s) rehabilitation may also positively
Unit of Prosthodontics and Crown and Bridge, Faculty of Dental Sciences, Institute of Medical Sciences, Banaras Hindu University, Varanasi, Uttar Pradesh, India

Corresponding Author: Atul Bhatnagar, Unit of Prosthodontics and Crown and Bridge, Faculty of Dental Sciences, Institute of Medical Sciences, Banaras Hindu University, Varanasi, India, Phone: +91 7080866678, e-mail: atuldent@hotmail.com

How to cite this article: Bhatnagar A. Long COVID-19: Rehabilitative and Restorative Challenges. Int J Prosthodont Restor Dent 2021;11(2):70.

Source of support: Nil

Conflict of interest: None

contribute to this. Shortened Dental Arch (SDA) treatment approach is a potential candidate of choice during the pandemic, delivering functional rather than non-essential treatments. ${ }^{9}$

Mobile dentistry may be extended to patients, in old-age homes or private homes, facing practical difficulties in movement and approaching a dental care service center/institution. ${ }^{6}$

A reassuring, caring, motivating demeanor on the part of the dental team, along with the aforementioned strategies, may meet the huge backlog of patients, treatment requirements of new as well as home/institution-bound patients, during these trying times.

\section{References}

1. "COVID-19 Dashboard by the Center for Systems Science and Engineering (CSSE) at Johns Hopkins University (JHU)"accessed on 14 August 2021.

2. 'https://www.ada.org/en/press-room/news-releases/2020-archives/ march/ada-calls-upon-dentists-to-postpone-elective-procedures/'.

3. The future of dentistry part 1: NHS Dr Catherine Rutland VOL 34 | ISSUE 6 | BDJ IN PRACTICE.

4. Surveys.ada.org/reports/RC/public/February 2021. ADA Health Policy Institute.

5. Prakash H, Chakrabarti A. Microorganisms 2021;9(3):523. DOI: 10.3390/ microorganisms9030523.

6. Aldhuwayhi S, Shaikh SA, Thakare AA, et al. Remote management of prosthodontic emergencies in the geriatric population during the pandemic outbreak of COVID-19. Front Med 2021;8:648675. DOI: 10.3389/fmed.2021.648675.

7. Watt RG. COVID-19 is an opportunity for reform in dentistry. Lancet 2020;396(10249):462. DOI: 10.1016/S0140-6736(20)31529-4.

8. Kathree BA, Khan SB, Ahmed R, et al. COVID-19 and its impact in the dental setting: a scoping review. PLoS ONE 2020;15(12):e0244352. DOI: 10.1371/journal.pone.0244352.

9. Nassani MZ, Shamsy E, Tarakji B, et al. Planning the restorative dental treatment at the time of coronavirus pandemic: a two-arm strategy. J Contemp Dent Pract 2021;22(1):1-3. 